\title{
TECNOLOGIA DOS NANOTUBOS DE CARBONO: TENDÊNCIAS E PERSPECTIVAS DE UMA ÁREA MULTIDISCIPLINAR
}

\author{
Marcelo Hawrylak Herbst* \\ Departamento de Física dos Sólidos, Instituto de Física, Universidade Federal do Rio de Janeiro, CP 68528, \\ 21945-970 Rio de Janeiro - RJ \\ Maria Iaponeide Fernandes Macêdo e Ana Maria Rocco \\ Departamento de Química Inorgânica, Instituto de Química, Universidade Federal do Rio de Janeiro, Cidade Universitária \\ 21945-970, Rio de Janeiro - RJ
}

Recebido em 3/11/03; aceito em 26/4/04; publicado na web em 27/8/04

\begin{abstract}
TECHNOLOGY OF CARBON NANOTUBES: TRENDS AND PERSPECTIVES OF A MULTIDISCIPLINARY AREA. An overview of the properties of carbon nanotubes is presented as a function of the structural characteristics and of the method of synthesis of these novel advanced materials. Emphasis is given to the catalytic decomposition of hydrocarbons over metal-supported catalysts and also the role of the support in obtaining homogeneous carbon nanotubes in high yelds is discussed. Some potential and real applications of carbon nanotubes are presented in a perspective view.
\end{abstract}

Keywords: carbon nanotubes; sol-gel; nanotechnology.

\section{INTRODUÇÃO}

A nanotecnologia vem despertando muito interesse nas comunidades científicas, e principalmente ao longo das últimas décadas muitos esforços foram feitos no sentido de atingir o tão desejado controle em nível atômico e molecular sobre os processos industriais. Com o surgimento dos materiais nanométricos, e em função deles, novas técnicas de caracterização foram projetadas e implementadas.

Um dos frutos desse interesse pelo domínio das pequenas dimensões foi a obtenção serendípica dos nanotubos de carbono (NC) sintetizados pela primeira vez em 1991 por Iijima ${ }^{1}$, usando o processo de pirólise de grafite em plasma sob atmosfera controlada de hélio. Apesar da contemporaneidade dessa descoberta, é curioso notar que já em 1889, numa patente norte-americana, foi relatado que filamentos de carbono podem ser formados a partir de hidrocarbonetos, em cadinhos de metal em altas temperaturas ${ }^{2}$.

Pode-se dizer que a descoberta de Iijima, em conjunto com os fulerenos descobertos por Kroto, Smalley e Curl em $1985^{3}$, abriu uma nova fronteira na química e na física do carbono.

A diversidade das aplicações, reais ou potenciais, dos NC, assim como a necessidade de controlar as morfologias apropriadas para sua utilização, fazem da pesquisa nesta área do conhecimento um trabalho de característica eminentemente multidisciplinar, envolvendo fatores que definem o sucesso de suas aplicações, tais como rota de síntese, processamento em formas variadas e qualidade dos NC.

O presente trabalho tem por objetivo mostrar as características principais dos NC, os métodos de síntese, purificação e isolamento, caracterização, manipulação e algumas aplicações desses novos materiais carbonosos. Em especial, a ênfase deste trabalho são os nanotubos de carbono de paredes múltiplas.

\section{Nanotubos de carbono}

Os NC são formados de arranjos hexagonais de carbono que originam pequenos cilindros. Eles usualmente têm uma faixa de di-

*e-mail: herbst@if.ufrj.br âmetro de poucos angstrons a dezenas de nanômetros e podem ter comprimento acima de vários centímetros ${ }^{4}$.

Do ponto de vista estrutural, há dois tipos de NC que podem apresentar alta perfeição $0^{5}$ os nanotubos de carbono de parede simples (NCPS), que podem ser considerados como uma única folha de grafite enrolada sobre si mesma para formar um tubo cilíndrico, e os nanotubos de carbono de parede múltipla (NCPM) que compreendem um conjunto de nanotubos concêntricos, num arranjo que lembra as tradicionais matrioshkas, bonecas russas ocas que trazem dentro outras bonecas ocas idênticas.

A maioria das propriedades dos NCPS depende do seu diâmetro e ângulo chiral, também chamado ângulo de helicidade, $\phi$. Estes dois parâmetros resultam dos chamados índices de Hamada $(\mathrm{n}, \mathrm{m})^{6}$.

Um NCPS pode ser construído a partir de uma folha de grafite enrolada de tal forma que coincidam dois sítios cristalograficamente equivalentes de sua rede hexagonal. $\mathrm{O}$ vetor $\mathbf{C}$ chamado chiral, que define a posição relativa dos dois sítios é definido mediante dois números inteiros $(n, m)$ e pelos vetores unitários da rede hexagonal $\mathbf{a}_{1}$ e $\mathbf{a}_{2}\left(\mathrm{C}=\mathrm{n} \mathbf{a}_{1}+\mathbf{m a}_{2}\right)$ como mostrado na Figura 1 .

Dependendo dos valores relativos do par $(n, m)$, os NCPS podem ser: armchair $(n=m)$, zig-zag $(n, m=0)$, ou chiral $(n \neq m \neq 0)$

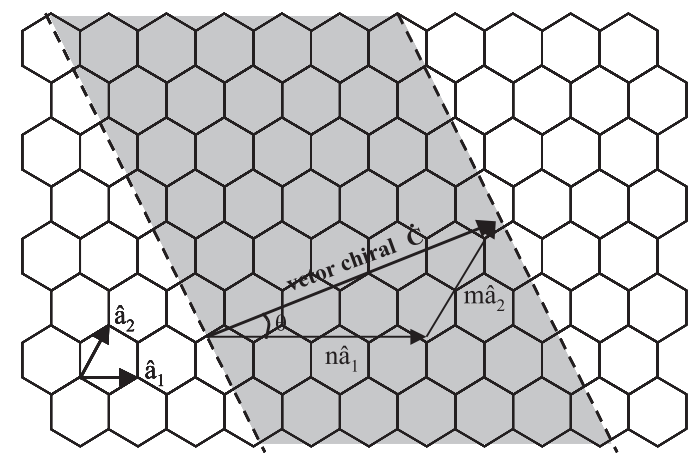

Figura 1. Diagrama da formação de nanotubos de carbono a partir de uma folha de grafite 
e estão definidos pelo ângulo de helicidade $\phi$. Se $\phi$ varia de 0 a $30^{\circ}$ podem ser gerados tubos partindo do tipo zig-zag até o armchair como limites, passando pelos tubos chiral. As três variedades de NCPS são ilustradas na Figura 2.
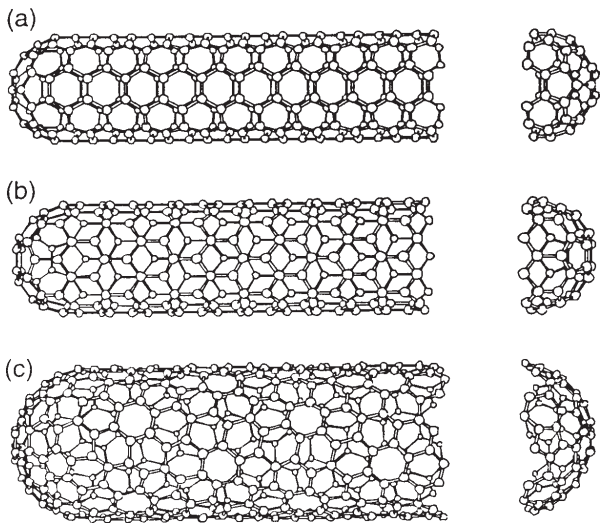

Figura 2. Geometrias de NCPS perfeitos: (a) armchair, (b) zig-zag, (c) chiral. Reproduzida da ref. 26 com permissão da Elsevier

\section{Propriedades eletrônicas dos nanotubos de carbono}

Os NCPS têm propriedades eletrônicas excepcionais. Em função dos índices de Hamada (n,m), um nanotubo é metálico quando n-m é multiplo de 3, em caso contrário é semicondutor. Todos os nanotubos do tipo armchair são metálicos, enquanto que os zig-zag e chiral podem ser metálicos ou semicondutores.

As propriedades eletrônicas de NCPM perfeitos assemelham-se às propriedades dos NCPS, por causa do fraco acoplamento entre os cilindros de carbono concêntricos. Além disso, por se tratarem de estruturas quasi-unidimensionais, o transporte eletrônico nos NC metálicos (tanto NCPS como NCPM) ocorre de forma balística, isto é, sem espalhamento, o que possibilita a condução de correntes através de grandes extensões do nanotubo sem aquecimento ${ }^{7}$.

Do ponto de vista das investigações teóricas, os NCPS representam os sistemas mais adequados, em relação aos NCPM, devido à maior facilidade na descrição computacional. Por outro lado, os NCPM são produzidos com mais facilidade e com menor custo que os NCPS.

\section{Métodos de síntese de NC}

Os métodos de síntese de NC empregados atualmente produzem somente uma pequena fração de NCPS, o que eleva em muito seu custo e praticamente impede sua aplicação em grande escala. Além do mais, no estágio atual, a maioria dos métodos de síntese gera grandes quantidades de impurezas.

Desde sua descoberta na fuligem originada pela pirólise de eletrodos de grafite em atmosfera controlada de hélio, os NC vêm sendo sintetizados também por outros métodos, como a síntese catalítica ${ }^{8}$, usando metais de transição sobre suportes de sílica, alumina e também sobre as zeólitas $\mathrm{Y}^{9}, \beta^{10}$ e ZSM- $5^{11}$, a deposição de vapor químico $(\mathrm{CVD})^{12}$, a decomposição de monóxido de carbono em altas pressões e altas temperaturas (o chamado processo HiPCO) ${ }^{13}$, e a erosão a laser ${ }^{14}$. Esses processos, bem como o processo original de pirólise do grafite apresentam o mesmo problema, a produção paralela de carbono amorfo e a presença inevitável de partículas metálicas oriundas dos catalisadores. No entanto, a síntese catalítica e a CVD possibilitam um maior controle das variáveis de síntese quando comparadas aos processos caóticos de pirólise em arco voltaico, erosão a laser e decomposição de monóxido de carbono. Portanto, com a otimização das condições de síntese tem-se demonstrado a possibilidade de obtenção de grandes quantidades de nanotubos de boa qualidade e com baixo teor de impurezas.

Se bem que a produção de NCPM conheça atualmente um estágio mais avançado, o que tem possibilitado algumas aplicações desses materiais, novos métodos de síntese, como o processo HiPCO, demonstram que há uma tendência crescente no sentido de se atingir o controle sobre a síntese em grande escala dos NCPS devido, principalmente, aos pequenos diâmetros associados a este tipo de NC e à sua estrutura eletrônica unidimensional, em alguns aspectos bastante distinta das propriedades dos NCPM.

\section{Formação de coque metálico e formação de NCPM}

Com relação à produção de NCPM usando catalisadores à base de suportes ácidos, é interessante notar que enquanto a pesquisa em catálise heterogênea usando metais de transição suportados é comumente dirigida para a prevenção da formação de depósitos de carbono (coque) na superfície do catalisador, essa característica indesejável nos processos catalíticos industriais pode ser aproveitada na obtenção de NCPM.

Catalisadores à base de metais de transição suportados são largamente usados no refino do petróleo e na indústria química e petroquímica, em um grande número de processos de relevância econômi$\mathrm{ca}^{15}$. Estudos sobre esse tipo de catalisador são importantes tanto do ponto de vista fundamental, isto é, da compreensão das reações microscópicas que ocorrem durante seu preparo, ativação e uso efetivo, quanto do ponto de vista de aplicações, seja na otimização e adequação a um determinado processo, seja no desenvolvimento de novos sistemas e processos catalíticos. É interessante notar que as propriedades desses catalisadores dependem fortemente do estado e da dispersão do componente metálico, que por sua vez são uma função do método de introdução do metal e do histórico da preparação do catalisador.

Com base na literatura ${ }^{8-12}$, no que diz respeito à produção catalítica de NCPM usando suportes ácidos, os metais preferencialmente estudados são o cobalto e o ferro, isolados ou em combinação, e como fonte de carbono são usados acetileno, propileno e etileno.

A escolha dos componentes metálicos e das fontes de carbono tem uma razão: é conhecido que catalisadores metálicos promovem a adsorção dissociativa e a desidrogenação de hidrocarbonetos, originando depósitos de carbono, por vezes de elevada cristalinidade, a temperaturas relativamente baixas e sem formação de quaisquer intermediários aromáticos policíclicos ${ }^{16}$. Todavia, a característica mais promissora dessa escolha é sem dúvida a morfologia dos depósitos de carbono, que pode se apresentar como filamentos, com partículas metálicas associadas, em geral no topo, por vezes ao longo do filamento.

Nesse sentido, o mecanismo mais aceito é o da difusão do carbono sobre a superfície e através do metal, como mostrado na Figura 3.

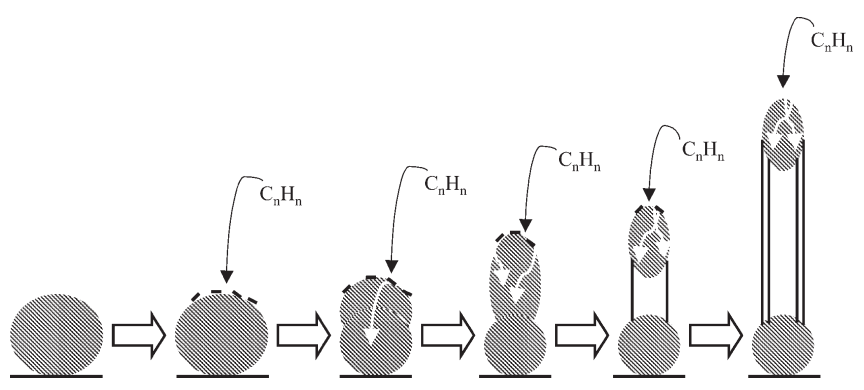

Figura 3. Esquema ilustrativo da formação de nanotubos de carbono segundo adaptação do mecanismo de crescimento de coque filamentar em catalisadores metálicos 
A partir do esquema apresentado na Figura 3, pode-se idealizar o seguinte mecanismo reacional para a formação catalítica de carbono filamentar, e numa extensão lógica, para a formação dos NCPM: (i) o hidrocarboneto é adsorvido e dissociado na superfície metálica, com formação de átomos de carbono adsorvidos; (ii) estes átomos de carbono podem dissolver-se e difundir-se através do metal, precipitando em regiões de crescimento preferencial, como as fronteiras de grão ou as interfaces metal/suporte. Desta forma, as partículas metálicas são destacadas da superfície e transportadas com os filamentos em crescimento, enquanto que a superfície ativa continua disponível para a reação já que o carbono não se acumula sobre ela; (iii) é também possível a nucleação à superfície, conduzindo à formação de filmes de carbono que efetivamente desativam o catalisador - o chamado carbono encapsulante.

Recentemente o mecanismo descrito acima foi confirmado por medidas de microscopia eletrônica de transmissão (TEM) in situ, realizadas em catalisador de $\mathrm{Ni} / \mathrm{SiO}_{2}$ sob aquecimento e submetido a uma atmosfera redutora de $\mathrm{H}_{2} / \mathrm{CH}_{4}{ }^{17}$.

É interessante notar que se a fonte de carbono for pobre em hidrogênio, como é o caso do acetileno, a formação de carbono filamentar e encapsulante é favorecida. Vale também lembrar que o mecanismo exposto acima depende fortemente da etapa de difusão/ precipitação, que só é favorecida para o caso dos metais que dissolvem facilmente o carbono e formam carbetos, como é o caso do $\mathrm{Fe}$, Co e Ni. A Figura 4 mostra o aspecto de NCPM obtidos pela decomposição catalítica do etileno sobre catalisadores de ferro, em que chama a atenção o alinhamento dos tubos de carbono.
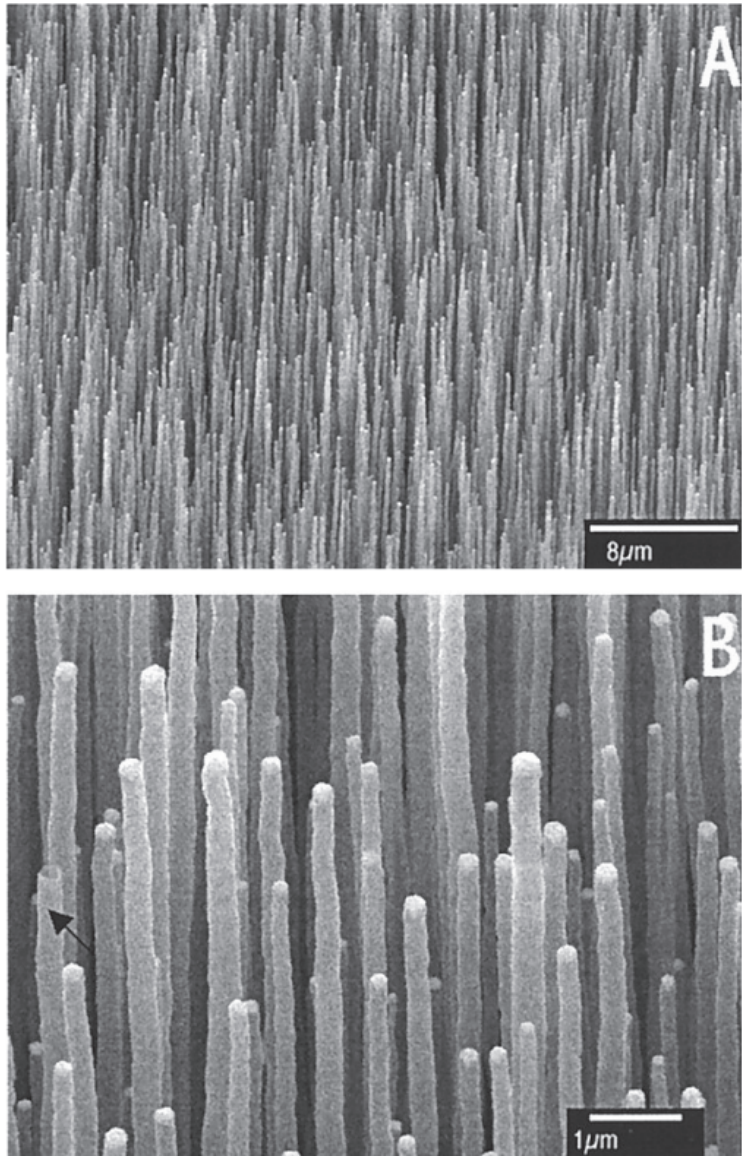

Figura 4. Microscopias eletrônicas de varredura de nanotubos de carbono obtidos por decomposição catalítica de etileno sobre catalisadores de ferro. Reproduzida de Chem. Mater. 1998, 10, 260 com permissão da American Chemical Society
Em trabalho recente, Piedigrosso e colaboradores ${ }^{11}$ mostraram que usando catalisador de cobalto suportado em sílica são produzidos principalmente nanotubos helicoidais, enquanto que o uso de alumina como suporte leva a uma distribuição bimodal de nanotubos, no que diz respeito ao diâmetro. Em ambos os casos foi produzida grande quantidade de carbono amorfo, diminuindo desta forma a qualidade dos NCPM. A observação constante de partículas metálicas no topo ou encapsuladas nos nanotubos sugere que o mecanismo descrito acima opera em ambos os casos. Além disso, a formação de depósitos de carbono amorfo reflete a formação de coque nãocatalítico, resultante de reações que envolvem como intermediários íons carbônio formados na superfície dos catalisadores em sítios ácidos de Brönsted ou Lewis ${ }^{16}$.

Alguns autores têm chamado a atenção para uma superioridade das zeólitas como suportes de partículas metálicas na obtenção de $\mathrm{NC}$, demonstrada pela produção de nanotubos de alta qualidade, isto é, praticamente sem defeitos, e com pequena quantidade de carbono amorfo como produto lateral ${ }^{9}$. Além do mais, o controle da acidez da componente zeólita do catalisador leva à diminuição substancial da produção do indesejável carbono amorfo, em contraste com os suportes sílica e alumina.

A introdução de etapas de pré-tratamento do catalisador, como o tratamento com vapor, também permite um controle mais fino sobre o diâmetro dos nanotubos obtidos quando o suporte é uma zeólita ${ }^{18,19}$.

\section{Efeito do método de introdução do metal no suporte}

A qualidade e o rendimento da produção dos NCPM depende fortemente do método de introdução do metal no suporte. Com efeito, em praticamente todos os relatos encontrados na literatura sobre a síntese catalítica de NCPM os catalisadores foram preparados pelos métodos clássicos, ou seja, impregnação, co-precipitação e troca iônica. É conhecido que cada um desses métodos de introdução do metal apresenta problemas quanto à homogeneidade das partículas metálicas formadas após as sucessivas etapas de preparação e, dessa forma, é necessário um controle mais apurado sobre a dispersão e principalmente sobre o tamanho das partículas metálicas. Vale notar que a tendência atual da área aponta para o uso de técnicas como solgel e deposição de vapor químico (CVD), com o objetivo de atingir esse controle ${ }^{7,20,21}$.

\section{Purificação dos nanotubos de carbono obtidos por síntese catalítica}

A purificação dos NCPM produzidos pela via catalítica é comumente feita em duas etapas: a) separação dos nanotubos do metal e do suporte e b) eliminação do carbono amorfo ${ }^{22-24}$. Na primeira etapa é utilizado um ácido mineral, tipicamente $\mathrm{HNO}_{3}, \mathrm{HF}$ ou $\mathrm{HCl}$, enquanto que a segunda etapa compreende oxidação do material carbonoso ao ar em temperatura elevada ou, alternativamente, oxidação por ácido nítrico ou permanganato de potássio em solução ácida, seguida de lavagem com água desmineralizada e filtração.

As zeólitas são facilmente atacadas e dissolvidas por ácidos minerais. Dessa forma elimina-se o suporte e as partículas metálicas do catalisador numa só etapa, de acordo com a equação

$$
\begin{aligned}
& \mathrm{Na}_{53} \mathrm{Al}_{53} \mathrm{Si}_{139} \mathrm{O}_{348}\left(\mathrm{H}_{2} \mathrm{O}\right)_{235}+768 \mathrm{HF} \rightarrow 139 \mathrm{SiF}_{4}+53 \mathrm{Al}^{3+}+53 \mathrm{Na}^{+}+ \\
& 212 \mathrm{~F}^{-}+619 \mathrm{H}_{2} \mathrm{O}
\end{aligned}
$$

(reação balanceada para a zeólita NaY, SAR 3) $)^{22}$

Por outro lado, a etapa da purificação que compreende a eliminação do carbono amorfo através da oxidação ao ar em temperaturas 
elevadas pode ser representada por

$\mathrm{C}+\mathrm{O}_{2} \rightarrow \mathrm{CO}_{2} \quad$ e $\quad 2 \mathrm{C}+\mathrm{O}_{2} \rightarrow 2 \mathrm{CO}$

Alternativamente, a oxidação por tratamento com solução ácida de permanganato de potássio pode ser descrita por

$3 \mathrm{C}+4 \mathrm{KMnO}_{4}+4 \mathrm{H}^{+} \rightarrow 4 \mathrm{MnO}_{2}+3 \mathrm{CO}_{2}+4 \mathrm{~K}^{+}+2 \mathrm{H}_{2} \mathrm{O}$

É interessante notar que os diferentes tratamentos levam comumente a diferentes morfologias dos nanotubos purificados, e que, portanto, deve-se otimizar a purificação de acordo com as características pretendidas para os NCPM.

\section{Caracterização dos nanotubos de carbono}

Na caracterização dos NC a técnica de escolha é a microscopia eletrônica de transmissão (TEM). Esta técnica mostra-se insubstituível no estudo da morfologia dos NC, bem como na detecção de partículas metálicas incorporadas aos nanotubos. Alguns exemplos significativos são mostrados na Figura 5.
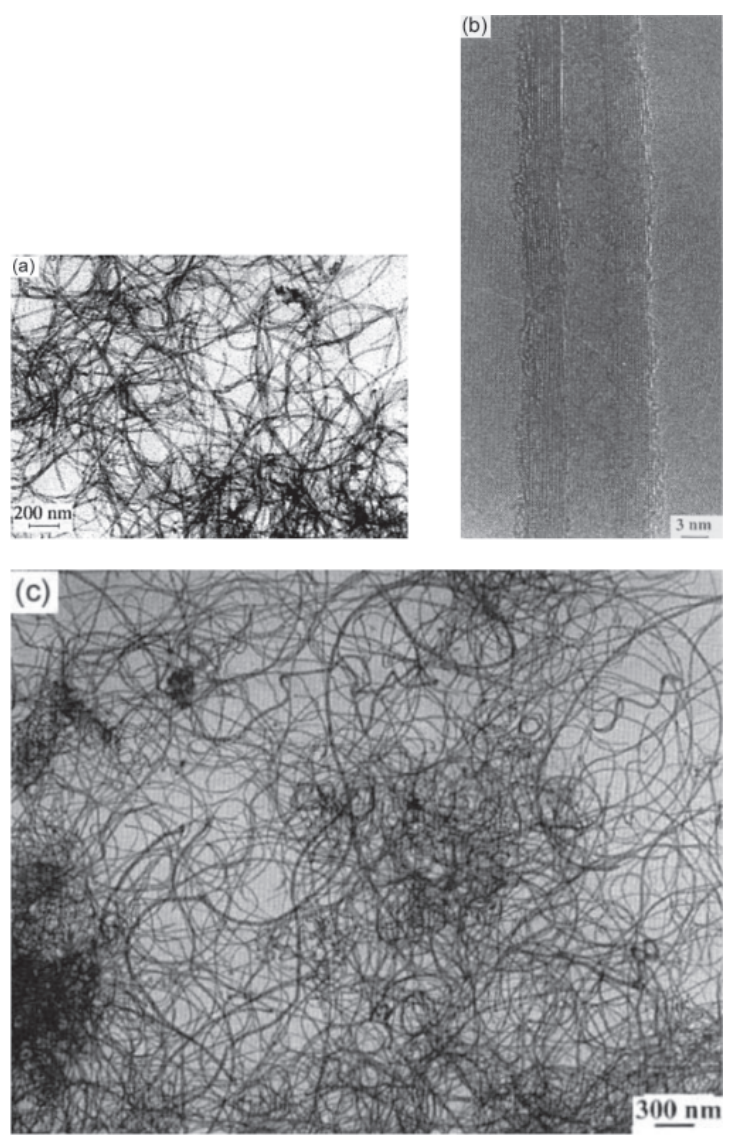

Figura 5. Imagens de nanotubos de carbono obtidas por TEM: (a) recémpreparados (reproduzida da ref. 22 com permissão de The Royal Society of Chemistry), (b) purificados por oxidação $\left(\mathrm{KMnO}_{4} / \mathrm{H}_{2} \mathrm{SO}_{4}\right)$, (c) HRTEM de NCPM. Reproduzida da ref. 11 com permissão de PCCP Owner Societies

Todavia, além da TEM outras técnicas mais rotineiras podem ser usadas para a caracterização dos NC, como as técnicas de caracterização textural, determinação de área superficial (BET), adsorção/ dessorção em temperaturas programadas (TPA/TPD) e porosimetria. Além dessas técnicas, o comportamento térmico dos NC pode ser estudado por técnicas termogravimétricas, como TGA e DSC. É interessante notar que os NCPM são mais estáveis em condições de altas temperaturas que os carvões amorfos e carvões ativados comumente usados como suporte na preparação de catalisadores. Essa característica diferenciada pode também ser usada na separação e purificação dos NCPM possibilitando, inclusive, estudos quantitativos com respeito à proporção NC/carbono não cristalino obtida nas diferentes condições experimentais de preparação.

Dentre as técnicas espectroscópicas empregadas na caracterização de fulerenos ${ }^{25}$ e nanotubos de carbono, a espectroscopia Raman é das mais valiosas. No caso dos NC, ela possibilita a diferenciação entre nanotubos, grafite e carbono amorfo. As bandas originadas por estruturas ordenadas, relacionadas a NC perfeitos são visualizadas em regiões bastante definidas no espectro Raman de uma amostra. Os modos vibracionais chamados de respiração radial ("radial breathing modes, RBM") são observados em baixos números de onda (entre 100 e $300 \mathrm{~cm}^{-1}$ ). Já os modos vibracionais chamados tangenciais são responsáveis pela chamada banda $\mathrm{G}$, próxima de $1575 \mathrm{~cm}^{-1}$, que é associada a nanotubos perfeitos. $\mathrm{A}$ banda $\mathrm{D}$, atribuída à presença de estruturas desordenadas, como nanotubos defeituosos e carbono não cristalino, é visualizada por volta de $1350 \mathrm{~cm}^{-1}{ }^{26}$. Além disso, a relação entre as áreas das bandas $\mathrm{G}$ e D, dada por $(1-\mathrm{D} / \mathrm{G})$ fornece o chamado 'parâmetro de qualidade', e reflete a proporção de nanotubos perfeitos numa determinada amostra. Na Figura 6 são mostrados segmentos de um espectro Raman típico de uma amostra de nanotubos de carbono com alta concentração de nanotubos perfeitos.

Entre outras técnicas espectroscópicas, a ressonância paramagnética eletrônica, RPE, em onda contínua ou pulsada, vem sen-

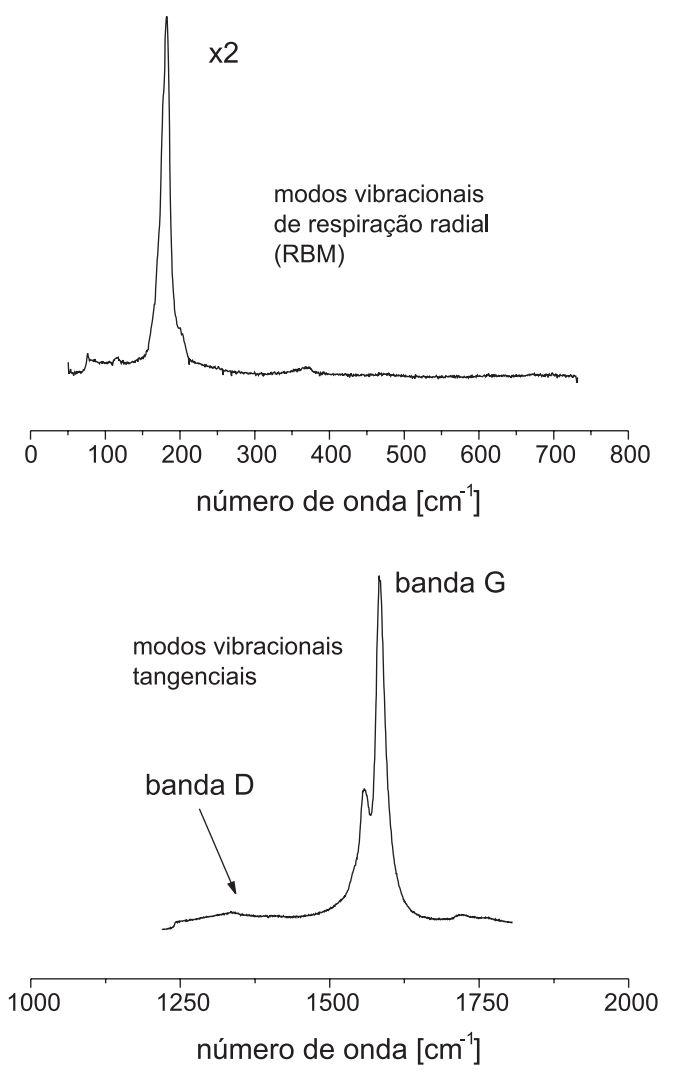

Figura 6. Espectro Raman típico de uma amostra de nanotubos de carbono. As regiões usuais e as bandas $G$ e $D$ estão indicadas (veja detalhes no texto). (Os autores agradecem à Dr. Vólia Lemos (IF-UFC) por permitir a divulgação dessas medidas) 
do usada na quantificação de defeitos, tanto intrínsecos à estrutura dos nanotubos (os chamados elétrons localizados) como extrínsecos, isto é, relacionados com a presença de carbono não cristalino (elétrons deslocalizados) ${ }^{27}$. Cada um desses defeitos apresenta linhas de ressonância em campos magnéticos distintos, possibilitando sua identificação e quantificação. Todavia, a presença do catalisador metálico, mesmo em baixa proporção, limita o uso desta técnica, devido ao fenômeno da ressonância ferromagnética, que apresenta linhas muito largas no espectro de RPE.

A espectroscopia de reflectância no infravermelho (IV) é uma técnica bastante difundida e bastante acessível, com resultados importantes para a caracterização de nanotubos de carbono, em especial no caso de amostras tratadas quimicamente, onde grupos funcionais podem estar ancorados em diferentes sítios, tanto internos como externos do nanotubo ${ }^{28}$.

Além dessas técnicas de caracterização, a microscopia eletrônica de varredura (SEM) é comumente usada em análises prévias, na verificação dos arranjos dos nanotubos sobre o suporte e, através da técnica de EDX, para o mapeamento dos diferentes componentes metálicos.

\section{Aplicações dos nanotubos de carbono}

Essa nova família de alótropos do carbono possui propriedades interessantes como alta resistência mecânica e capilaridade, além de apresentar estrutura eletrônica única, apontando para diversas aplicações no futuro. De fato, muitas aplicações potenciais para os NC foram propostas, incluindo a obtenção de compósitos condutores ou de alta resistência mecânica, dispositivos para armazenamento e conversão de energia, sensores, dispositivos semicondutores em escala nanométrica, entre outras. De um ponto de vista de aplicações diretas, os NC poderiam ser ainda usados como peneiras moleculares, como material para armazenamento de hidrogênio, como aditivos para materiais poliméricos e como suporte em processos catalíticos, por exemplo. Algumas dessas aplicações encontram-se atualmente em fase comercial, ao passo que outras estão ainda em fase de testes, como discutido criticamente num artigo de revisão publicado na revista Science ${ }^{7}$. Em particular, a utilização dos NC para armazenamento de hidrogênio é tema ainda controverso ${ }^{29}$. Atualmente, o alto custo, a falta de controle sobre o processo (tipo, comprimento e diâmetro do nanotubo formado) e limitações ao processo de purificação constituem sérios obstáculos para a maioria das aplicações dos NC.

A seguir serão descritas em maior detalhe algumas aplicações dos NC.

\section{Aditivos para materiais poliméricos}

As propriedades excepcionais dos nanotubos têm motivado muitas pesquisas acerca das propriedades mecânicas de compósitos à base de nanotubos. Nesse sentido, a dispersão uniforme ao longo da matriz polimérica e a otimização tanto da solubilidade nanotubo/ matriz como da adesão são parâmetros críticos. Em especial, a dispersão é um ponto crítico para o reforço de materiais poliméricos, uma vez que os NC encontram-se comumente agrupados em feixes. Todavia, existem relatos de que a adição de pequenas porcentagens de NC a matrizes poliméricas como PVA ou PS levam a um aumento de cerca de $40 \%$ na tensão elástica e a um acréscimo de aproximadamente $25 \%$ na resistência à tração $0^{30}$. Em outras palavras, a performance dos materiais compósitos de nanotubos de carbono é cerca de 10 vezes melhor que o observado para compósitos das fibras de carbono convencionais, para uma mesma carga. Um ponto que chama a atenção é o desenvolvimento de técnicas que possibilitem um 'ancoramento' efetivo dos nanotubos à matriz polimérica.
Nesse sentido, as modificações químicas dos nanotubos representam uma alternativa promissora. Cabe ressaltar que seriam desejáveis estudos de modelagem e simulação das propriedades dos compósitos poliméricos à base de NC.

\section{Materiais adsorventes de gases}

O desenvolvimento de sistemas para a remoção dos óxidos de nitrogênio $\mathrm{NO}_{\mathrm{x}}$ das emissões originadas pela queima de combustíveis fósseis é um tema bastante atual, principalmente por causa da questão ambiental. O principal objetivo desse tipo de pesquisa é encontrar adsorventes adequados, isto é, que possam liberar NO, seja pelo aumento da temperatura ou pela diminuição da pressão, para a conversão em $\mathrm{N}_{2}$. Nesse sentido, atualmente os materiais mais promissores são os carvões ativados e funcionalizados com grupos $\mathrm{FeO}(\mathrm{OH})$. No entanto, recentemente foi relatado na literatura ${ }^{31}$ que $\mathrm{NC}$ possuem propriedades superiores na adsorção de $\mathrm{NO}_{x}$, especialmente em condições de baixas pressões parciais, onde os materiais convencionais apresentam desempenho pouco satisfatório.

\section{Aplicações biotecnológicas}

O objetivo desses estudos é a imobilização de proteínas e enzimas, etapa fundamental para o desenvolvimento de tecnologias de biosensores e bioreatores ${ }^{32,33}$. Com efeito, nanotubos de carbono foram utilizados para a imobilização de metalotioneína $\left(\mathrm{Zn}_{2} \mathrm{Cd}_{5}\right)$ extraída de fígado de coelho, citocromo $\mathrm{C}$ (extraído de coração de cavalo) e $\beta$-lactamase, extraído de Bacillus cereus ${ }^{34}$. As amostras foram analisadas por TEM e apontam para a imobilização das proteínas no interior dos NC, na forma de monômeros, dímeros e oligômeros. Como esperado, ocorreu uma seleção por tamanho no encapsulamento das proteínas. É interessante notar que uma parcela dos nanotubos apresenta condutividade metálica, característica que em combinação com a seletividade por tamanho e com a forte interação proteína/suporte aponta para biosensores potenciais ${ }^{35}$.

\section{Adsorventes de metais pesados em efluentes}

NC apresentam capacidade excepcional e alta eficiência de adsorção na remoção de chumbo de meio aquoso. A adsorção é influenciada pelo $\mathrm{pH}$ da solução e também pela presença ou não de grupos funcionais na superfície dos NC, que pode ser controlada por tratamentos químicos e térmicos. Resultados publicados recentemente sugerem que $\mathrm{NC}$ são bons adsorventes para $\mathrm{Pb}^{2+}$ e têm grande potencial na área de remediação ${ }^{36}$.

\section{Armazenamento de hidrogênio}

O objetivo principal dessas pesquisas é o desenvolvimento de um material de baixo custo para armazenamento de hidrogênio, com capacidade maior que $5 \%$ em peso, segundo normas do Departamento de Energia do Governo dos Estados Unidos ${ }^{37}$. Além disso, esse material deve ser estável ao longo da ciclagem de hidrogênio e possuir características termodinâmicas e cinéticas adequadas tanto para o transporte como para o desenvolvimento de dispositivos portáteis ${ }^{29}$, os quais poderiam ser aplicados para mover veículos com células a combustí$\mathrm{vel}^{38}$. A tecnologia dos nanotubos de carbono representa uma nova fronteira para o armazenamento de hidrogênio, especialmente se esses materiais puderem ser modificados para armazenar grandes quantidades de hidrogênio à temperatura ambiente. É interessante notar que o meio mais promissor para o armazenamento de hidrogênio são os NCPS, difíceis de serem obtidos em grandes quantidades, mas cujas modificações químicas são bastante promissoras. Naturalmente isso 
não exclui os estudos voltados para a avaliação das propriedades de armazenamento dos NCPM.

\section{Utilização como suporte em catalisadores}

Nos últimos anos, pesquisas relacionadas aos NC têm mostrado que esses materiais apresentam comportamentos diferenciados e não usuais na área de catálise, em comparação com os materiais clássicos. Nesse sentido, a produção de estireno industrial a partir da desidrogenação catalítica do etilbenzeno representa um exemplo. Essa reação é comumente catalisada por ferro suportado em óxidos, ocorrendo mesmo assim em altas temperaturas e na presença de excesso de vapor. Todavia, várias complicações, como a formação de coque e limitações termodinâmicas tornam essa rota muito dispendiosa. Uma rota alternativa é a desidrogenação oxidativa, que emprega temperaturas mais baixas e não apresenta limitações termodinâmicas. Alguns relatos sugerem que o carbono é a espécie ativa neste processo, mas a baixa estabilidade dos catalisadores à base de carbono ativado em presença de agentes oxidantes é o fator limitante para o desenvolvimento desses materiais. Nesse sentido, e devido à alta estabilidade térmica dos NC, esses materiais vêm sendo utilizados como suporte. Recentemente foi verificado que NC preparados por síntese catalítica $\left(\mathrm{Ni} / \mathrm{Al}_{2} \mathrm{O}_{3}\right.$ ou $\left.\mathrm{Fe} / \mathrm{SiO}_{2}\right)$ apresentam alto rendimento em estireno e boa estabilidade nas condições operacionais da reação de desidrogenação oxidativa ${ }^{39}$.

\section{Funcionalização dos nanotubos de carbono}

A manipulação e processamento dos nanotubos de carbono individuais é limitada pela sua insolubilidade. Todavia, recentemente foram obtidos bons resultados com o chamado 'recobrimento' ("wrapping") dos NC com polímeros, levando inclusive à obtenção de arranjos supramoleculares nanotubo-polímero ${ }^{40}$. Apesar de escassos, os relatos sobre este tópico apontam para a modificação química dos NC, o que pode encurtar o caminho até as aplicações, incluindo a preparação de compósitos. Essas modificações orgânicas englobam longas cadeias de alquilas, polímeros, reações de radicais, entre outras, que possibilitaram a obtenção de nanotubos funcionalizados tanto lateral como longitudinalmente, eventualmente levando a materiais com boa solubilidade ${ }^{41}$

Além dessas funcionalizações orgânicas, existe um relato de funcionalização organometálica, a reação de NC com o complexo de Vaska, trans-[( $\left.\left.\mathrm{PPh}_{3}\right)_{2} \operatorname{Ir}(\mathrm{CO}) \mathrm{Cl}\right]$, que produz adutos solúveis em solventes orgânicos ${ }^{42}$. Tanto $\mathrm{NC}$ recém-preparados quanto purificados por oxidação foram tratados com o complexo de Vaska, e foi verificado que o centro metálico de Ir coordena-se aos nanotubos de duas formas distintas: nos nanotubos recém-preparados, a coordenação ocorre de maneira $\eta^{2}$, ao passo que nos nanotubos oxidados a reação procede através dos átomos de oxigênio presentes na superfície do tubo, formando um complexo hexacoordenado de Ir. Dessa forma, a funcionalização de NC representa um desafio ao químico sintético, quer sejam testados grupos orgânicos como organometálicos.

\section{Nanotubos de carbono no Brasil}

Nos últimos anos vêm sendo realizadas atividades na área de nanotubos de carbono no Brasil. Para se ter uma idéia do desenvolvimento desta área em nosso país, recentemente os NC foram tema de artigo de capa da revista Ciência Hoje: Nanotubos e a nova era do carbono $^{43}$. Na área de síntese de $\mathrm{NC}$, entre outros gupos, destacam-se o grupo de C. A. Luengo (UNICAMP), que dispõe de reatores a plasma de grafite, adequados à produção de NCPS, o grupo de
L. O. Ladeira (UFMG), que desenvolve pesquisas utilizando a técnica de CVD e o grupo de A. Zarbin (UFPR), que utiliza precursores organometálicos "single-source". No campo da caracterização dos NC, destacam-se o grupo de M. Pimenta e A. Jório (UFMG), e os grupos de P. Corio (USP-SP), V. Lemos e J. Mendes Filho (UFC), que realizam estudos sobre as propriedades eletrônicas dos NC utilizando a espectroscopia Raman.

A funcionalização dos $\mathrm{NC}$ vem sendo pesquisada pelo grupo de $\mathrm{F}$. O. Plentz Filho (UFMG), além de outros pesquisadores que realizam estudos teóricos, como M. S. Mazzoni (UFMG), R. Mota (UFSM), R. Baierle (Unifra-RS), A. J. R. da Silva e A. Fazzio (USP). Ainda no campo teórico, mas com ênfase na chamada nanomecânica, o grupo de R. Capaz e B. Koiller (UFRJ) vem desenvolvendo interessante trabalho.

Mais recentemente iniciou-se no Grupo de Materiais Condutores do Instituto de Química da Universidade Federal do Rio de Janeiro projeto que visa a implantação de técnicas de produção, purificação e funcionalização de NC. O enfoque inicial do grupo de estudos é a utilização de sólidos ácidos como suportes para a produção de nanotubos de carbono, que serão posteriormente utilizados na fabricação de compósitos ou como componentes de pilhas a combustível.

\section{CONCLUSÕES}

A descoberta dos $\mathrm{NC}$, há pouco mais de uma década, alavancou as pesquisas no campo da chamada nanotecnologia e trouxe a perspectiva de uma nova etapa na tecnologia eletrônica, na qual a miniaturização dos dispositivos será uma das conseqüências, indo da escala micrométrica para a nanométrica.

Seguramente ainda existe um longo caminho para se alcançar as metas de redução de custos e aumento da eficiência na produção de NC. Além disso, o controle sobre as propriedades e características estruturais dessas nanoestruturas é ainda muito rudimentar. Há, todavia, um desenvolvimento sensível dos processos, no sentido de tornar rotineiras as técnicas de produção de NC. Além do mais, como já destacado por outros autores, umas das características da chamada nanociência é seu rápido avanço. Dessa forma, considerando-se o presente estágio de desenvolvimento tecnológico/científico, a crescente interação entre Universidade, Institutos de Pesquisa e Empresas, além das iniciativas de financiamento público e privado, podese esperar que muitas das aplicações propostas para os NC, além de outras a surgir, serão realizadas.

\section{AGRADECIMENTOS}

M. H. Herbst e M. I. F. Macêdo agradecem ao CNPq pelas bolsas de pesquisa.

\section{REFERÊNCIAS}

1. Iijima, S.; Nature 1991, 354, 56.

2. Hughes, T. V.; Chambers, C. R.; U.S. pat. 405,480 1889.

3. Kroto, H. W.; Heath, J. R.; O’Brien, C. O.; Curl, R. F.; Smalley, R. E.; Nature 1985, 318, 162.

4. Day, L.; Mau, A. W. H.; Adv. Mater. 2001, 13, 899.

5. Dresselhaus, M. S.; Dresselhaus, G.; Eklund, P. C.; Science of Fullerenes and Carbon Nanotubes, Academic Press: San Diego, 1996.

6. Hamada, N.; Sawada, S.; Phys. Rev. Lett. 1992, 68, 1579.

7. Baughman, R. H.; Zakhidov, A. A.; de Heer, W. A.; Science 2002, 297, 787.

8. Ivanov, V.; Nagy, J. B.; Lambin, Ph.; Lucas, A.; Zhang, X. B.; Zhang, X. F.; Bernaerts, D.; Van Tendeloo, G.; Amelinckx, S.; Van Landuyt, J.; Chem. Phys. Lett. 1994, 223, 329.

9. Hernadi, K.; Fonseca, A.; Nagy, J. B.; Bernaerts, D.; Fudala, A.; Lucas, A. A.; Zeolites 1996, 17, 416. 
10. Kamalakar, G.; Hwang, D. W.; Hwang L-P.; J. Mater. Chem. 2002, 12, 1819.

11. Piedigrosso, P.; Konya, Z.; Colomer, J-F.; Fonseca, A.; Van Tendeloo, G.; Nagy, J. B.; Phys. Chem. Chem. Phys. 2000, 2, 163.

12. Li, W. Z.; Xie, S. S.; Qian, L. X.; Chang, B. H.; Zou, B. S.; Zhou, W. Y.; Zhao, R. A.; Wang, G.; Science 1996, 274, 1701.

13. Chiang, I. W.; Brinson, B. E.; Huang, A. Y.; Willis, P. A.; Bronikowski, M. J.; Margrave, J. L.; Smalley, R. E.; Hauge, R. H.; J. Phys. Chem. B 2001, 105, 8297

14. Thess, A.; Lee, R.; Nikolaev, P.; Daí, P.; Petit, P.; Robert, J.; Xu, C.; Lee, Y. H.; Kim, S. G.; Rinzler, A. G.; Colbert, D. T.; Scuseria, G. E.; Tomanek, D.; Fisher, J. E.; Smalley, R. E.; Science 1996, 273, 483.

15. Minachev, Kh. M.; Isakov, Ya. I. Em Zeolite Chemistry and Catalysis; Rabo, J. A.; ed.; American Chemical Society: Washington, 1976, cap. 10.

16. Figueiredo, J. L.; Ribeiro, F. R.; Catálise Heterogénea, Fundação Calouste Gulbenkian: Lisboa, 1987, cap. 7.

17. Helveg, S.; López-Cartes, C.; Sehested, J.; Hansen, P. L.; Clausen, B. S.; Rostrup-Nielsen, J. R.; Abild-Pedersen, F.; Norskov, J. K.; Nature 2004, 427, 426.

18. Zhang, A.; Li, C.; Bao, S.; Xu, Q.; Microporous Mesoporous Mater. 1999, $29,383$.

19. Willems, I.; Kónya, Z.; Colomer, J-F.; Van Tendeloo, G.; Nagaraju, N.; Fonseca, A.; Nagy, J. B.; Chem. Phys. Lett. 2000, 317, 71.

20. Lee, C. J.; Park, J.; Carbon 2001, 39, 1891.

21. Hou, H.; Schaper, A. K.; Weller, F.; Greiner, A.; Chem. Mater. 2002, 14, 3990.

22. Colomer, J-F.; Piedigrosso, P.; Willems, I.; Journet, C.; Bernier, P.; Van Tendeloo, G.; Fonseca, A.; Nagy, J. B.; J. Chem. Soc., Faraday Trans. 1998, 94, 3753.

23. Colomer, J-F.; Piedigrosso, P.; Fonseca, A.; Nagy, J. B.; Synth. Met. 1999, $103,2482$.

24. Lobach, A. S.; Spitsina, N. G.; Terekhov, S. V.; Obraztsova, E. D.; Phys. Solid State 2002, 44, 475.

25. Herbst, M. H.; Pinhal, N. M.; Demétrio, F. A. T.; Dias, G. H. M.; Vugman, N. V.; J. Non-Cryst. Solids 2000, 272, 127.
26. Eklund, P. C.; Holden, J. M.; Jishi, R. A.; Carbon 1995, 33, 959.

27. Lu, K. L.; Lago, R. M.; Chen, Y. K.; Green, M. L. H.; Harris, P. J. F.; Tsang, S. C.; Carbon 1996, 814.

28. Kuhlmann, U.; Jantoljak, H.; Pfänder, N.; Journet, C.; Bernier, P.; Thomsen, C.; Synth. Met. 1999, 103, 2506.

29. Kajiura, H.; Tsutsui, S.; Kadono, K.; Kakuta, M.; Ata, M.; Murakami, Y.; Appl. Phys. Lett. 2003, 82, 1105.

30. Thostenson, E. T.; Ren, Z.; Chou, T. -W.; Composites Sci. Technol. 2001, $61,1899$.

31. Long, R. Q.; Yang, R. T.; Ind. Eng. Chem. Res. 2001, 40, 4288

32. Wagner, P.; FEBS Lett. 1998, 430, 112.

33. Moser, J.; Naughton, M.; NNUN Abstracts 2002 Materials, Physics, Processes \& Characterization, 1972.

34. Davis, J. J.; Green, M. L. H.; Hill, H. A. O.; Leung, Y. C.; Sadler, P. J.; Sloan, J.; Xavier, A. V.; Tsang, S. C.; Inorg. Chim. Acta 1998, 272, 261.

35. Sloan, J.; Cook, J.; Green, M. L. H.; Hutchison, J. L.; Tenne, R.; J. Mater. Chem. 1997, 7, 1089.

36. Li, Y. -H.; Wang, S.; Wei, J.; Zhang, X.; Xu, C.; Luan, Z.; Wu, D.; Wei, B.; Chem. Phys. Lett. 2002, 357, 263.

37. Zidan, R.; Proceedings of the 2001 DOE Hydrogen Program Review, NREL/CP-570-30535.

38. Dillon, A. C.; Jones, K. M.; Bekkedahl, T. A.; Kiang, C. H.; Bethune, D. S.; Heben, M. J.; Nature 1997, 386, 377.

39. Keller, N.; Maksimova, N. I.; Roddatis, V. V.; Schur, M.; Mestl, G.; Butenko, Y. V.; Kuznetsov, V. L.; Schögl, R.; Angew. Chem., Int. Ed. 2002, $41,1885$.

40. Georgakilas, V.; Kordatos, K.; Prato, M.; Guldi, D. M.; Holzinger, M.; Hirsh, A.; J. Am. Chem. Soc. 2002, 124, 760, e referências.

41. Lin, Y.; Rao, A. M.; Sadanadan, B.; Kenik, E. A.; Sun, Y. -P.; J. Phys. Chem. B 2002, 106, 1294, e referências.

42. Banerjee, S.; Wong, S. S.; Nano Lett. 2002, 2, 49.

43. Capaz, R. B.; Chacham, H.; Ciência Hoje 2003, 33, 20. 